\title{
Escenarios distópicos en la literatura colombiana*
}

Recibido: 31/08/2018 | Revisado: 01/11/2018 | Aceptado: 23/11/2018

DOI: $10.17230 /$ co-herencia.16.30.1

\author{
Clemencia Ardila J.**
}

aardila@eafit.edu.co

\begin{abstract}
Resumen La literatura colombiana no ha sido ajena a imaginar escenarios futuros; a conjeturar acerca de cómo el espacio geográfico de nuestras ciudades se transforma, producto de nuevos modelos de organización social y política; a predecir el destino de los colombianos de los años o siglos venideros, a trazar la cartografía del país del mañana. Particularmente, en los primeros años del 2000 se publican dos novelas: El cerco de Bogotá (2003) de Santiago Gamboa e Iménez (2011) de Luis Noriega, que focalizan su atención en Bogotá y, desde propuestas genéricas diferentes, configuran universos posibles de valencia negativa. Los escenarios propuestos por estos dos autores, así como las relaciones con el poder, la ciencia y la literatura misma, son algunos de los motivos temáticos que se analizan en este trabajo.
\end{abstract}

\section{Palabras clave:}

Literatura colombiana, distopía, ciencia ficción, novela policiaca, Santiago Gamboa, El cerco de Bogotá, Luis Noriega, Iménez.

\section{Dystopian scenarios in Colombian literature}

\begin{abstract}
Colombian literature has not been alien to imagining future scenarios, to conjecture how the geographical space of our cities is transformed into new forms as a result of new social and political organization models, to predicting the destiny of Colombians in the centuries to come and to mapping the country of tomorrow. Two particular novels were published in the early 2000's, El cerco de Bogotá (2003) by Santiago Gamboa, and Iménez (2011) by Luis Noriega, which focus on Bogota and shape, from different genres, possible universes of negative valence. This paper aims to analyze the scenarios proposed in these two works as well as a variety of thematic motifs, including power structures, science, and literature itself.
\end{abstract}

\section{Keywords:}

Colombian literature, dystopia, science fiction, police novel, Santiago Gamboa, El cerco de Bogotá, Luis Noriega, Iménez.
Este trabajo es resultado de la investigación "Índice de Villanos", enero-diciembre de 2018, código 851-000003, inscrita en el grupo de investigación "Estudios en filosofía, hermenéutica y narrativas" de la Universidad EAFIT.,

** Doctora en Literatura de la Universidad de Antioquia, Colombia. Profesora-investigadora de la Universidad EAFIT, Medellín - Colombia ORCID: 0000. 0002- 9458-9877. 
En el último libro del sociólogo polaco Zygmunt Bauman, Retrotopía (2017), encontramos una afirmación acerca de la literatura de ciencia ficción que, además de llamar nuestra atención, nos inquietó. Pero antes de citarla, es importante presentar su contexto: Bauman demuestra, en las primeras páginas de su libro, cómo nuestra época se caracteriza por una percepción absolutamente negativa acerca del futuro. Es esta una visión en la que predomina la desconfianza y el terror a muchas posibles situaciones; entre otras, enumera las siguientes:

El terror a perder el trabajo y el estatus social asociado a este, el terror a que nos confisquen el hogar y el resto de nuestros bienes y enseres, el terror a contemplar impotentes cómo nuestros hijos caen sin remedio por la espiral descendente de la pérdida de bienestar y prestigio, y el terror a ver las competencias que tanto nos costó aprender y memorizar despojadas del poco valor de mercado que les pudiera quedar (Bauman, 2017, pp. 15-16).

Las condiciones laborales, la propiedad privada, la familia y la profesión están en riesgo de pérdida, expropiación o desuso. El panorama no puede ser más desolador.

Esta percepción, como bien lo demuestra Bauman, la comparten otros sociólogos y filósofos, ${ }^{1}$ y también los artistas: "nuestras películas y novelas de ciencia ficción se catalogan cada vez más a menudo dentro de las secciones de cine de terror y literatura gótica" (Bauman, 2017, p. 61).

¿Por qué resultan inquietantes estas palabras? Porque nos hablan de los rasgos que quizá caracterizan hoy en día la literatura y el cine de ciencia ficción. Nos dicen que las acompaña el propósito de generar, en el lector, el miedo, la zozobra y el espanto, producto de unas acciones signadas por la violencia, por lo desconocido o, simplemente, por lo inesperado. Nos señalan cómo, en un giro hacia el pasado de la historia literaria, se configuran escenarios sórdidos y oscuros, ambientes sombríos y tenebrosos, por donde transitan personajes

1 Entre muchos otros, Bauman (2017, pp. 11-21) cita a Rutger Bregman y sus estudios sobre utopía para realistas; los de Javier Solana, sobre el impacto, en Estados Unidos, de prácticas que pretenden un retorno a un modelo del pasado; el diagnóstico sobre las formas de la nostalgia, de la profesora de literatura Svetlana Boym, que signan nuestra época y, en fin, una amplia lista de filósofos, sociólogos y literatos. 
signados por la maldad y dotados de poderes extraordinarios — que no sobrenaturales-.

Pero estas palabras dicen mucho más: nos hablan de la pérdida de la esperanza y la confianza en los tiempos por venir; de la visión negativa de escritores y creadores ficcionales; de la falacia que para muchos representa ese adagio popular que pregona que "vendrán tiempos mejores"; indican, en suma, una concepción del futuro, en el que este ya no se avizora como un tiempo de estabilidad — política, social, económica-, sino, por el contrario, como la entronización de la violencia y el caos y, lo más importante, como el momento en que los derechos del ciudadano del común a una vida digna, estable y tranquila, desaparecerán definitivamente.

A este diagnóstico de nuestra época parecen hacer eco dos novelas de la literatura colombiana, El cerco de Bogotá (2003) de Santiago Gamboa e Iménez (2011) de Luis Noriega, obras que le apuestan a imaginar cómo será Colombia en general y en particular la capital, Bogotá, en los próximos siglos: como un territorio signado por la guerra (Gamboa) o por una estratificación social de orden económico y demográfico (Noriega). Novelas en la que se proponen diversos mecanismos de control sobre el ciudadano del común, sutiles las más de las veces, explícitamente violentos y coercitivos otros, con el fin de favorecer a una clase privilegiada y preservar un Estado capitalista. Obras, en fin, en las que predomina una visión del porvenir según la cual, como bien lo dice Bauman, el futuro "ha dejado de ser el hábitat natural de las esperanzas y las más legítimas expectativas para convertirse en un escenario de pesadillas" (2017, p. 15).

La forma que toman tales pesadillas, las acciones de quienes las hacen posibles, la ciudad como lugar de acogida del terror y el desasosiego son, entre otros, los motivos temáticos presentes en las novelas de Gamboa y Noriega que a continuación se analizan, con el fin de trazar la cartografía del futuro que se imagina y conjetura desde estas dos obras de la literatura colombiana.

Estas dos novelas conjeturan acerca de la ciudad del mañana, de la capital de mediados o finales los siglos XXI y XXIII, y comparten una visión negativa del futuro y, en esa medida, tienen un carácter distópico, esto en atención a las definiciones más comunes del género (Braga, 2006) según las cuales se contrapone a las ficciones utópicas, 
que proponen un universo ficcional de valencia positiva. Pero, valga la aclaración, estas novelas se distancian en términos genéricos, pues mientras en Iménez se pueden observar motivos temáticos propios de la ciencia ficción, como avances tecnológicos e informáticos, por nombrar uno de los más comunes, la novela de Gamboa tiene una vocación policiaca y es a sus reglas a las que se acoge su trama.

Nos interesa, entonces, más allá de las clasificaciones genéricas, analizar los escenarios geográficos, políticos y sociales del que, según estos dos escritores, podrían ser nuestro futuro. Cada uno de los apartados en que se divide este trabajo se dedica a este propósito.

\section{Escenario de guerra}

En El cerco de Bogotá se nos propone un futuro para Colombia signado por la violencia, la destrucción de su capital y la inseguridad de sus ciudadanos. En el siglo XXI, en una fecha que el narrador no precisa,${ }^{2}$ la guerra civil, entre las fuerzas del Estado, la guerrilla y los paramilitares, es ya una realidad. Bogotá, la capital, es el epicentro de la lucha armada, y su dominio, el objetivo a alcanzar.

La guerrilla ocupa el sur y los cerros orientales, y controla el tráfico de drogas y licor; los paramilitares dominan la zona occidental y el aeropuerto El Dorado, por lo que el ingreso al país está bajo su potestad; el Ejército conserva el norte y el centro de la ciudad, y el hotel Tequendama es el baluarte donde se concentran los corresponsales de guerra y se emiten las comunicaciones oficiales.

El Gobierno central y algunos ciudadanos, para preservar sus vidas, se trasladan a la zona Atlántica del país; desde allí, el presidente y sus ministros diseñan las estrategias para reconquistar la ciudad.

Este es el contexto general en el que su autor propone una trama de corte policiaco, en la que dos periodistas buscan esclarecer la participación de hombres del Ejército, como socios de la guerrilla, en el tráfico de armas y de cocaína.

2 No hay mayores indicios al respecto en la novela; solo esta anotación permite precisar el siglo XXI como el tiempo de la historia: "En el fragor de los incendios, las llamas fueron tan altas que algunos bogotanos recordaron lo ocurrido el 9 de abril de 1948, esa memorable fecha de destrucción a mediados del siglo anterior" (2003, p. 15). 
La Bogotá del siglo XXI se describe como sigue:

La plaza de Bolívar era un terreno baldío repleto de cráteres y escombros, pues ahí la lucha había sido fuerte. El Capitolio Nacional, el Palacio de Justicia y la Casa de Nariño mostraban sus vientres reventados. La gente, en un acto de suma desesperanza, había saqueado lo que quedaba de las antiguas oficinas, de los amplios salones y aforos. En sus ruinas, los mendigos se protegían de la lluvia y del frío haciendo fuego con viejos legajos. El gobierno, al principio de la guerra, intentó defender la sede histórica y esto avivó la destrucción. Llovieron bombas desde las montañas, hubo atentados ciegos, se ametralló sin piedad el centro de la ciudad hasta que no quedó ni un alma (2003, p. 15).

En este fragmento resultan interesantes algunas de las estrategias del narrador para presentar esa ciudad del futuro. Inicialmente, focaliza su atención en la Plaza de Bolívar, centro del poder y lugar de fundación de la ciudad. A su alrededor se sitúan los edificios que albergan las diferentes ramas del gobierno y se despliegan los diferentes barrios de la ciudad.

La enumeración considera tres elementos: el territorio mismo y sus edificaciones; los ciudadanos y, por último, las acciones gubernamentales. Del primero se resalta cómo ha perdido su función inicial de simbolizar al Estado y su poder, para convertirse en otro signo más de la guerra. Es un territorio baldío que, además de contener las ruinas de un esplendor pasado, no detenta oficio ni utilidad alguna.

En esa misma dirección se presentan los edificios que albergan los tres poderes del sistema político colombiano: legislativo, judicial y ejecutivo, y se homologan con un cuerpo vivo que expone, sin pudor alguno, sus entrañas, y que, a cambio de magistrados, ministros y congresistas, hospeda solo a mendigos (la que parece ser la clase social más baja en la escala de valores del narrador). Para culminar este cuadro de desolación, se presentan los ciudadanos como saqueadores y las acciones del Gobierno como erráticas y causantes de mayor violencia y destrucción.

En este escenario geográfico vive una sociedad fragmentada en clases sociales, según su adscripción a ideologías de derecha o izquierda, gobernada por un sistema político democrático, pero endeble y corrupto. Militares, guerrilleros, paramilitares y periodistas son traficantes y negocian por igual alimentos y el cuerpo de las 
mujeres, armas e información, drogas y salvoconductos, y, por supuesto, con la vida y la dignidad de los pocos habitantes obligados por las circunstancias a permanecer en la ciudad.

Sobresalen, en este escenario, los protagonistas de la novela, dos corresponsales extranjeros que, al igual que sus colegas, también acuden al soborno, a las drogas y otras estratagemas para dirimir el enigma de una situación cuyo resultado, a decir verdad, por lo común que resultan sus ingredientes, es bastante predecible para cualquier lector, sea o no colombiano.

Como puede observarse en esta breve presentación, la novela extrema las circunstancias y las situaciones del conflicto armado de los últimos años en Colombia; retoma sus protagonistas y desplaza, temporalmente - pocos o muchos años, no se sabe, pero para el caso da lo mismo-, unos hechos que hacen parte de nuestra historia. En otros términos, esta novela, primero, radicaliza una situación política y social para crear un mundo indeseable y, segundo, realiza una crítica política y social del contexto colombiano de los últimos años.

Tal crítica se dirige a mostrar cómo militares, guerrilleros, paramilitares y periodistas son todos corruptos y, además, comparten su interés en incrementar sus arcas y satisfacer sus deseos personales (estatus social, económico, o simplemente éxito profesional). Son esos los únicos principios que defienden, por encima de una postura ideológica y política. Se denuncia, entonces, la primacía de tales intereses por parte de los grupos políticos de nuestra sociedad, y se señala la corrupción como un rasgo propio de la idiosincrasia colombiana, la del presente y la del futuro. Pero la que podría ser la crítica más importante, esto es, cómo no existen principios ideológicos sobre los cuales fundamentar y justificar un enfrentamiento bélico y, por supuesto, un modelo de organización política y social, se diluye en medio de la reproducción de estereotipos de vieja data, tanto sobre el papel del hombre y la mujer en nuestra sociedad, como del corresponsal de guerra.

Este narrador, como se demostró en otra ocasión (Ardila-Jaramillo, 2006), replica, en el primer caso, discursos machistas y, en el segundo, visiones idílicas sobre la guerra, en la que esta se convierte, para el periodista, en un escenario para la aventura y se le despoja del horror de la muerte, de la destrucción y de la desolación. Así, la 
advertencia acerca de la inminencia de la catástrofe que significa continuar por la vía del conflicto armado se queda en el decorado, en señalar algunas de las características de sus protagonistas, los cuales comparten los mismos vicios, las mismas ideas, los mismos objetivos. Pero ¿no hay acaso, tras ese escenario tan homogéneo, otra posibilidad? ¿Acaso corresponde al lector configurarla? El alto grado de referencialidad de la novela, justifica la ausencia de una postura crítica y la representación banalizada de una situación signada por la violencia y la corrupción?

Las palabras del también novelista Javier Cercas, cuando habla de la responsabilidad del escritor y los límites de su ética como elementos que confluyen en la ficción con el propósito de dar cuenta de las que denomina "relaciones sutiles e inextricables entre lo real y lo inventado" (2017, p. 12), señalan un camino diferente al elegido por Gamboa en esta novela. Un camino en el que antes que el reduccionismo, se privilegian las diferentes aristas de la problemática del conflicto armado en Colombia para dar cuenta de su complejidad y, por supuesto, de las consecuencias de continuar en tal estado de cosas.

En síntesis, y como conclusión de la lectura de esta primera novela, habría que decir que a la visión distópica que ofrece y el escenario futuro que propone para Colombia, le falta la voz del poeta; de aquel que, como bien lo dice Ricœur (2006), da cuenta de una experiencia de mundo, sí, pero cuya configuración como mundo del texto está signada por una redescripción del mundo del ser humano, por proponer otros modos de comprender la realidad y nuestra relación con los otros y con nosotros mismos.

Así lo hace uno de nuestros poetas, Juan Manuel Roca, cuya voz resulta pertinente en este momento. Dice:

Sin saber para quién,

Escribo esta carta puesta en el buzón del viento,

Desde una nación donde alguien proscribe el sueño,

Donde gotea el tiempo como lluvia envilecida

Y la risa es condenada por traición a los espejos.

No sé a quién pedirle que abra su ventana (2016, p. 86). 


\section{Escenario totalitario}

Iménez (2011), la novela de Luis Noriega, propone un escenario futuro donde un sistema político y social, omnipotente y omnipresente, niega la libertad de los ciudadanos, impone fronteras geográficas, restriñe la movilidad de sus habitantes a ciertos espacios e impide cualquier amago de sublevación. Esta presentación general de la novela podría describir muchas otras de naturaleza distópica, en las cuales también se conjetura un panorama plagado de violencia y desesperanza para la humanidad. Este hecho, antes que señalar una falencia en la obra de Noriega, habla de la presencia de algunos tópicos - un Estado dictatorial, violencia y negación de la libertad, la ciudad como escenario distópico—, propios del género, en su objetivo de configurar un mundo signado por valencias negativas.

La trama de la novela gira en torno a la vida de su protagonista, Iménez, quien narra un episodio de su vida, 20 días según se marca en cada capítulo, a manera de un diario para dar cuenta de una serie de sucesos que transforman su percepción de la sociedad en la que vive. El escenario es Ciudad Andina, de la que precisa el narrador en el paratexto que precede a la historia, en una suerte de fórmula matemática para definir una variable: "sea una ciudad cualquiera". Con esta aclaración, quizá se pretende trascender los límites geográficos del espacio de la ficción y llamar la atención sobre cómo ese mundo posible bien podría ubicarse fuera de los límites del país. Sin embargo, los nombres de algunos de los barrios, Chicó Oriental, Santafé, Santo Domingo, señalan referencialmente hacia la capital.

El siguiente episodio, en el que Iménez narra las peripecias a las que se enfrenta para llevar un gato a su casa y poder disfrutar de una cena gourmet, bien puede servir para trazar las características de la ciudad y de la sociedad del siglo XXIII que se proponen en esta novela. Dice:

Llevar al gato a casa fue más complicado de lo que imaginé. Sin máscara para el pobre animal era inútil intentar el subterráneo, y conseguir otra clase de transporte para abandonar Ciudad Andina es bastante difícil. [...] Detuve sin éxito seis deslizadores [...] los afiliados no pueden salir de la Cúpula y los residentes autorizados a circular por el exterior suelen negarse o cobran recargos prohibitivos. Resuelto a no irme sin 
mi gato, opté por abandonar la Cúpula a pie y buscar algo afuera. Fue la pesadilla que había previsto. Tan pronto estuvimos a cielo abierto las ratas empezaron a seguirnos. Para evadirlas, decidí salir de la carretera circunvalar y atravesar el antiguo cordón sanitario. Tenía la esperanza de encontrar transporte en Santo Domingo, pero dos francotiradores autorizados a disparar primero y preguntar después estuvieron cerca de matarme en cuanto intenté refugiarme en los niveles superiores. Terminamos en Santafé sin haber perdido a las ratas y, como ya había tenido que hacer en el cordón sanitario, saqué el incinerador portátil y cociné un par que se acercaron demasiado. [...] Corrí tres o cuatro bloques hasta encontrar un acceso seguro a la autovía y con ayuda del coprometabolizador detuve el primer vehículo que pasó y me hice llevar a casa (2011, pp. 27-28).

El fragmento da cuenta de varias de las características de la imaginada metrópoli del siglo xxIII y de algunos de los motivos temáticos dominantes de la novela. En primer lugar, los cambios son evidentes en la ciudad, en su atmósfera, en el entorno geográfico. El principal, la mutación de las ratas, cuyo número y ferocidad son considerables, persiguen a los hombres y asolan la ciudad, y ante las cuales humanos y animales son impotentes. La única defensa son las armas y un gas venenoso para los ciudadanos, que se esparce por el subterráneo como único medio para ahuyentarlas. La ciencia y la tecnología han unido esfuerzos para crear nuevos medios de transporte, como los deslizadores; pero también para inventar nuevas armas: un incinerador portátil y un coprometabolizador, arma está que, como se revela páginas más adelante, reduce a materia fecal a un cuerpo.

Todos estos cambios, antes de denotar progreso y desarrollo, muestran un deterioro ambiental significativo. Y es en esta dirección hacia donde se dirige ese primer llamado de atención de la novela y se cumple ese propósito admonitorio asignado a la literatura distópica (Martín-Rodríguez, 2011, p. 157).

Las acotaciones donde su protagonista se lamenta y acusa por el mundo que le tocó en suerte son significativas; dice, por ejemplo:

Nací tarde. El profesor Groot no llegó a conocer las vacas. Yo no alcancé ni a los perros. En este sentido, los ejecutores somos una víctima más de un error cometido por los exploradores de Ganímedes (Noriega, 2011, p. 22). 
Además del exterminio de esas dos especies tan preciadas por el hombre, por proveer alimentación y compañía, el fragmento atribuye a sus antepasados, científicos y gobernantes, la responsabilidad no solo de los desastres ambientales que, según se enuncia en la novela, son de gran magnitud, sino también del papel que le ha tocado en suerte desempeñar: es un ejecutor. Es uno de los empleados de Determinación de Vacantes, dependencia del Ministerio de Inteligencia, encargada, como su nombre lo indica, de gestionar la liberación de apartamentos y puestos de trabajo, hecho para el cual deben morir quienes los ocupan. En síntesis, son, como bien lo expresa uno de ellos, quienes "proveen servicios fúnebres a domicilio" (2011, p. 17).

Las palabras del personaje, entonces, van más allá de señalar a los culpables del exterminio de algunos animales y del dominio de las ratas (que se han comido a los perros); también denuncian cómo el desastre ambiental es una de las causas por las cuales el Estado se arroja el derecho a ejercer un control demográfico, a determinar qué mujeres pueden o no concebir un hijo, a limitar la vida de algunos de sus ciudadanos a 45 años.

Por supuesto, los gobernantes se escudan en su obligación de garantizar la supervivencia de la especie humana y aducen que se trata de un "aprovechamiento racional de la fuerza laboral disponible" (Noriega, 2011, p. 23); pero tras esta razón se oculta un interés económico y de clase: velar por los privilegios y las comodidades de la clase dirigente, los residentes, mantener un sistema capitalista, asegurar una estratificación jerárquica y económica. Así lo denuncia uno de los manifiestos del movimiento "Muerte a Residentes" que, a manera de epígrafe, se presenta en la segunda parte de la novela:

Apuntalados por esa cantera inagotable de mano de obra esclava que se extiende más allá de las fronteras de la Cúpula, los residentes han construido un régimen de opresión que con cinismo han llamado "sistema de privilegios" (Noriega, 2011, p. 55).

Tal "sistema de privilegios" constituye el fundamento de la organización social y de la distribución del espacio de Ciudad Andina, segundo motivo temático mediante el cual se traza la cartografía del escenario distópico que propone Noriega. 
La sociedad está divida en dos grandes grupos: los que viven en la Cúpula y los del exterior. Los primeros pueden ser residentes o afiliados; los del exterior son los ciudadanos del común, traficantes, prostitutas, vendedoras de óvulos, renegados y los ejecutores.

Los residentes viven en la Cúpula, una especie de paraíso, donde sus habitantes están libres del peligro que representan las ratas y disfrutan de comodidades vedadas a los ciudadanos del común, y al que no se puede entrar sino con una insignia que identifica el ADN del usuario. Son los residentes quienes ocupan los altos cargos del gobierno y de las empresas privadas; es a ellos a quienes corresponde decidir sobre la vida y la muerte de las otras clases de ciudadanos.

La categoría de "afiliados" se obtiene por un contrato con el Estado para acceder a algunos de los privilegios de los residentes. La edad mínima para ser un afiliado es 19 años y el contrato finaliza a los 45, edad en la que debe morir.

En el exterior, la inseguridad, la basura, las privaciones, los peligros acechan a sus habitantes, aquellos cuya capacidad económica no les permite comprar su ascenso a residentes, aquellos que se niegan a ser afiliados porque su origen se los impide (son fruto de nacimientos ilegales), o bien por ser opositores al régimen y hacen parte de diferentes movimientos de resistencia.

Otro de los rasgos de una narración distópica consiste en hacer de una sociedad real su referente y el mundo posible de la obra "se presenta en relación dialéctica” (Martín-Rodríguez, 2011, p. 155) con aquella. A semejanza de la novela de Gamboa, en este caso, también, en la organización social y política del mundo de la novela se escuchan los ecos de nuestra sociedad actual; pero a diferencia de aquella, se problematizan sus rasgos. Para el lector de hoy no son extrañas las fronteras guardadas por francotiradores ni tampoco la inversión de los procedimientos: disparar primero y preguntar después. El sistema político imperante en Ciudad Andina también es similar: un presidente, ministros, congresistas y un largo séquito de funcionarios públicos que velan por sus propios intereses y no atienden a los de los ciudadanos. Un sistema, además, avalado por "La Sociedad de Naciones", por la "Unión Caribe" y hasta por las colonias en Marte y Calíope. 
Existe, sin embargo, una diferencia —algunos podrían calificarla de aparente y con razón-, a saber: el Estado ha ideado un sistema de control demográfico que le otorga el poder sobre la vida y la muerte. Los esfuerzos del Gobierno, como se denuncia en la novela, se dirigen a perfeccionar los métodos de esterilización de las mujeres afiliadas y las armas de los ejecutores, y no a mejorar las condiciones de vida de los ciudadanos del "exterior". Bien lo sintetiza el personaje en el siguiente diagnóstico y balance de su sociedad, cuando se pregunta: “Qué era entonces lo que el mundo necesitaba conocer?", y responde, citando las palabras del profesor Groot, oponente al sistema, así:

Las miserias de la vida cotidiana, los modos de existencia anómalos que ha creado el sistema de privilegios, las amarguras de los afiliados y de todos los que están al servicio de los afiliados, las vendedoras de óvulos de Garcés Navas, las compradoras de óvulos de la Cúpula, los políticos convertidos en traficantes de aplazamientos, los médicos convertidos en mercaderes de un sistema fundado en la negación de la vida, los publicistas de Ingresos y Reacomodación, los reducidores de Dotación y, por supuesto, ustedes, los amigos de Determinación de Vacantes (Noriega, 2011, p. 24).

Ese es, en síntesis, el escenario del siglo XXIII que se configura en la novela de Noriega, un escenario plagado de miserias, de modos de sobrevivir que, paradójicamente, suponen renunciar a la vida misma, negarse a procrear y aceptar una "fecha de caducidad" (Noriega, 2011, p. 40), como bien lo expresa uno de los personajes; una existencia, en fin, que deja un sabor amargo en sus habitantes.

Un escenario, además, en el que los destrozos ambientales, producto de acciones irresponsables, o por lo menos, equivocadas por parte de la ciencia y el Gobierno, ha trastocado el comportamiento de las personas, a tal punto que la vida misma es una mercancía, en todas sus etapas, desde la gestación hasta la muerte.

En la Bogotá del futuro se incrementarán exponencialmente las diferencias, económicas, educativas, laborales, entre las distintas clases sociales; la imposición de fronteras, ya no invisibles, al interior de una misma ciudad, será un hecho; los ciudadanos perderán el derecho a la libre expresión, a un trabajo y a una vida digna; los mecanismos de control por parte de la clase dominante serán más sofisticados y refinados — pastillas, incineradores portátiles para 
agilizar el trámite de la muerte, insignias con lector de ADN para controlar los movimientos de los ciudadanos externos, cárceles y centros de rehabilitación ciudadana-, y el sistema de gobierno tendrá la omnipotencia que muchos hoy desean. En suma, un escenario que resulta a todas luces poco deseable.

Pero en esta obra de Noriega resulta interesante no solo la proyección hacia un futuro, también llama la atención el movimiento del siglo XXIII hacia el pasado. Un pasado que es nuestro presente y hacia el cual se torna la mirada del personaje, para advertir sobre las consecuencias de acciones y políticas sociales, ambientales, científicas, gubernamentales irresponsables y, al tiempo, da cuenta de una postura ideológica que propugna por un retorno a ciertos principios éticos y de convivencia social propios, algunos de nuestra época, otros, quizás, de quienes nos antecedieron.

Que tal intención de relación dialéctica con la sociedad del presente acompaña la configuración del mundo posible de la novela, se evidencia en otro de sus motivos temáticos dominantes: la literatura. Uno de sus personajes, o para ser más precisos, uno de los afiliados, a quien debe atender el ejecutor Iménez, es un escritor cuya saga sobre el "ejecutor Jaramillo" es todo un éxito en ventas. Sus novelas, al igual que la que tiene entre manos el lector, cuenta las intimidades del día a día del oficio de los llamados "cocineros" de seres humanos; de sus relaciones amorosas, de los sentimientos y las posturas éticas que lo llevan a actuar contra la ley y, en fin, relatan de qué manera puede, así sea por una sola ocasión, vengarse del sistema.

En ese sentido, el día cinco de la novela se revela como su ars poética y en un movimiento metaficcional ${ }^{3}$ ofrece al lector la clave de su organización en tres partes, cada una de las cuales se dedica a los temas antes relacionados y, por supuesto, enuncia una concepción particular acerca de la literatura.

3 La metaficción se define, en términos generales, como aquella modalidad literaria que hace de la ficción su tema y, por tanto, en el desarrollo de la trama se exponen y revelan los artificios del proceso de creación de una obra. De esta manera, se evidencia el carácter ilusorio y ficcional de la literatura. Un estudio amplio sobre el tema lo desarrollo en el libro El segundo grado de la ficción: estudio sobre los procesos metaficcionales en la narrativa colombiana contemporánea (Vallejo, Abad Faciolince y Jaramillo Agudelo) (2014). 
En esa dirección, afirma, por boca del escritor ficcionalizado, que "toda novela ambiciosa es una reescritura de la historia" (Noriega, 2011, p. 44), y que su propósito no es, como podría pensarse, develar los misterios de la vida humana, sino "crear un misterio donde en realidad no hay ninguno, que es un sustituto como cualquier otro" (Noriega, 2011, p. 45). Tales afirmaciones resultan significativas e invitan a leer una novela que conjetura un posible futuro de nuestra sociedad, como otra forma de considerar nuestro presente y nuestro pasado, pero que, de igual manera, reconoce los alcances limitados, por múltiples razones, de la literatura misma en su empeño, si no de transformar, por lo menos de proponer otro modo de concebir la vida, de habitar el mundo, de relacionarnos con los otros.

Para concluir la lectura de esta novela, es oportuno señalar que también hace eco a otra de las afirmaciones de Bauman sobre la tendencia a retornar a "mundos ideales ubicados en un pasado perdido/robado/abandonado que, aun así, se ha resistido a morir" (Bauman, 2017, p. 14). Diremos entonces, sirviéndonos de algunas palabras de este sociólogo, que ese topos, ese territorio, esa ciudad que se imagina y configura en un tiempo por venir, se moldea a partir de una mirada retrospectiva, producto de una reflexión crítica que no busca un "retorno directo a un modo de vida que se practicara con anterioridad", lo cual es imposible, sino la revalorización de algunos aspectos del pasado que, desde una perspectiva presente, se consideran "demostradamente validados e indebidamente abandonados o imprudentemente descuidados" (Bauman, 2017, p. 18). ¿Cuáles? La libertad, el respeto a la vida, la conservación del medio ambiente y, sobre todo, la esperanza en un futuro mejor. Así, Noriega, al momento de imaginar, predecir, conjeturar un futuro para el hombre, muestra no una, sino dos caras del mismo asunto: su futuro y su pasado; aquello que quizá suceda y lo que equivocadamente se dejó atrás. Quizá esta sea la vocación última de la literatura distópica. 


\section{Conclusiones}

Como se enunció en las páginas iniciales de este trabajo, las novelas de Gamboa y de Noriega se inscriben en dos géneros diferentes desde los cuales proponen una visión de la sociedad colombiana del mañana, pero mientras de Iménez puede afirmarse que es futurista y anticipatoria, como conviene a la ciencia ficción, El cerco de Bogotá no puede calificarse como tal, pues su intención de configurar un mundo posible situado en el futuro no tiene pretensiones diferentes a las que los cánones del género policiaco delimita. Así, el llamado de Noriega resulta muy diferente del de Gamboa, pues si bien ambos escritores conjeturan a partir de nuestro presente un futuro distópico para Colombia, cada uno de ellos lo hace desde perspectivas muy diferentes. Esta diferencia, antes que un obstáculo, se ofrece como una alternativa para resaltar que, más allá de las fronteras de los géneros, la literatura es el espacio para la conjetura, la anticipación y, sobre todo, para la problematización de todo aquello que conforma nuestra sociedad, nuestra cultura y, por supuesto, nuestra humanidad.

Para Gamboa, el conflicto armado, con sus actores y dinámicas, es el único elemento a tener en cuenta, y si bien es innegable su incidencia en la organización social y política del país, también es cierto que es uno entre otros factores a considerar. En esa dirección, la obra de Noriega ofrece una conjetura más extensa (en amplitud y profundidad), al llamar la atención sobre las tres variables, ecología, ciencia y política, de esa ecuación acerca del futuro de Colombia, de América Latina y del mundo, aún por resolver, pero cuyos términos hacen parte de nuestros escenarios pasados y presentes.

Ya lo había dicho la poeta polaca Wislawa Szymborska:

Cuando pronuncio la palabra Futuro, la primera sílaba pertenece ya al pasado $(2014$, p. 394)【 


\section{Referencias}

Ardila-Jaramillo, C. (2006). Las mudanzas de la identidad. Medellín: Editorial Universidad EAFIT.

Ardila-Jaramillo, C. (2014). El segundo grado de la ficción: estudio sobre los procesos metaficcionales en la narrativa colombiana contemporánea (Vallejo, Abad Faciolince y Jaramillo Agudelo). Medellín: Editorial Universidad EAFIT.

Bauman, Z. (2017). Retrotopía. Barcelona: Paidós.

Braga, C. (2006). Utopie, eutopie, dystopie et anti-utopie. Metabasis, año I (2), 3-20. Recuperado de http://www.metabasis.it/articoli/2/2_braga.pdf.

Cercas, J. (2016). El punto ciego. Las conferencias Weidenfeld 2015. Bogotá: Ramdon House.

Gamboa, S. (2003). El cerco de Bogotá. Barcelona: Ediciones B.

Martín-Rodríguez, M. (2011). La ciudad libertaria del futuro en la distopía El amor dentro de 200 años (1932), de Alfonso Martínez Rizo. Ángulo recto, 3(2), 151-169. Recuperado de http://revistas.ucm.es/index.php/ANRE/article/view/37582

Noriega, L. (2011). Iménez. Bogotá: Taller de edición Roca.

Ricœur, P. (2006). Del texto a la acción. Ensayos de hermenéutica 1I. Buenos Aires: Fondo de Cultura Económica.

Roca, J. M. (2016). Silabario del camino. Poesía reunida 1973-2014. Medellín: Confiar y Letra a Letra.

Szymborska, W. (2014). Poesía no completa. México: Fondo de Cultura Económica. 